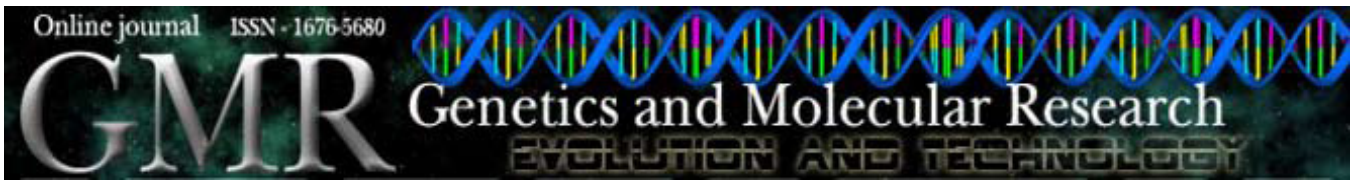

\title{
An interspecific QTL study of Drosophila wing size and shape variation to investigate the genetic basis of morphological differences
}

\author{
B.P. Matta and B.C. Bitner-Mathé \\ Departamento de Genética, Instituto de Biologia, \\ Universidade Federal do Rio de Janeiro, Rio de Janeiro, RJ, Brasil \\ Corresponding author: B.C. Bitner-Mathé \\ E-mail: bcbitner@biologia.ufrj.br
}

Genet. Mol. Res. 9 (4): 2032-2049 (2010)

Received May 21, 2010

Accepted July 22, 2010

Published October 13, 2010

DOI 10.4238/vol9-4gmr929

\begin{abstract}
The Drosophila wing has been used as a model in studies of morphogenesis and evolution; the use of such models can contribute to our understanding of mechanisms that promote morphological divergence among populations and species. We mapped quantitative trait loci (QTL) affecting wing size and shape traits using highly inbred introgression lines between $D$. simulans and $D$. sechellia, two sibling species of the melanogaster subgroup. Eighteen QTL peaks that are associated with 12 wing traits were identified, including two principal components. The wings of $D$. simulans and $D$. sechellia significantly diverged in size; two of the QTL peaks could account for part of this interspecific divergence. Both of these putative QTLs were mapped at the same cytological regions as other QTLs for intraspecific wing size variation identified in D. melanogaster studies. In these regions, one or more loci could account for intra- and interspecific variation in the size of Drosophila wings. Three other QTL peaks were related to a pattern of interspecific variation in wing size and shape traits that is summarized by one principal component. In addition, we observed that
\end{abstract}


female wings are significantly larger and longer than male wings and the second, fourth and fifth longitudinal veins are closer together at the distal wing area. This pattern was summarized by another principal component, for which one QTL was mapped.

Key words: Quantitative variation; QTL mapping; Transgressive segregation; Drosophila simulans; Drosophila sechellia; Drosophila melanogaster

\section{INTRODUCTION}

One approach to help understand the processes that promote morphological evolution is to determine the genetic basis of quantitative traits. This could help clarify some related matters, such as how genetic variation is maintained in natural populations, the mechanisms that cause populations and species to diverge, and whether the infinitesimal model appropriately describes the genetic control of quantitative traits (Falconer and Mackay, 1996).

The Drosophila wing has been used as a model in studies on morphogenesis and evolution (Lecuit and Le Goff, 2007), and the adaptive nature of quantitative variation in wing size has been supported by various of these studies. Recurrent clinal variation in size has been observed within species; wings are generally larger at higher latitudes or altitudes, which are both colder environments (Bitner-Mathé and Klaczko, 1999a; Gilchrist et al., 2000; Huey et al., 2000). Increased flight performance in response to temperature has been considered as a functional explanation for this phenomenon (Gilchrist and Huey, 2004). However, since many ectotherms show an adaptive thermal response, with larger bodies and body parts usually found at lower temperatures (Atkinson and Sibly, 1997), these wing size clines could be due to a correlated response to selection on body size or growth rate. Consequently, at least as a proxy for body size, wing size can be considered an adaptive trait (Gilchrist and Partridge, 2001). This is not as clear for quantitative variation in wing shape.

Adaptive or not, any trait that varies within and between species is clearly of interest from an evolutionary perspective. But little is known about the genetics of interspecific variation in Drosophila wings: interspecific studies are less common and, to our knowledge, no attempt has been made to identify the loci that contribute to between-species variation. For such a study, the melanogaster group provides a suitable model system. Some species can be intercrossed to yield fertile hybrids, allowing standard mapping of quantitative trait loci (QTL). Besides, specialization is expected to have occurred relatively rapidly for species of this group. This should be the case for $D$. sechellia, which is endemic to some islands of the Seychelles arquipelago and breeds in the fruit of Morinda citrifolia, a toxic resource for other insects (Louis and David, 1986). Drosophila sechellia differs in a number of physiological and behavioral traits when compared to closely related species, such as the cosmopolitan generalists D. simulans and D. melanogaster (Jones, 2005). Since adult morphology is directly influenced by larval processing of nutritional resources (Bochdanovits and De Jong, 2003), it is possible that interspecific variation in wing size and shape might be achieved through selection and adaptation of $D$. sechellia to morinda fruit. However, D. sechellia has reduced molecular variation compared to its sibling species (Hey and Kliman, 1993), which indicates a possible founder event and a role of drift 
in early phenotypic divergence.

Unraveling the genetic basis of interspecific differences would allow investigation of whether wing traits and the respective genes have evolved through drift or natural selection within this group. However, to date, the knowledge regarding which genes are related to quantitative variation in Drosophila wings comes from D. melanogaster studies. For instance, in mutational screenings via $P$-element insertions, up to 68 and 43 genes were found to affect the quantitative variation of wing size (Carreira et al., 2009) and shape components (Weber et al., 2005; Dworkin and Gibson, 2006), respectively. Even though a candidate approach could be used to test if some of these genes also contribute to phenotypic divergence among close species, choosing the candidates seemed rather tentative. And, as recently reviewed by Mackay et al. (2009), segregating variation in natural populations (and in different species) is not necessarily maintained at the loci required for wild-type expression of the trait.

So, we took advantage of classic QTL mapping to find chromosome regions that enclose one or more loci involved with the genetic control of interspecific variation in Drosophila wings. More specifically, our goals were to map QTLs associated with wing size and shape traits in highly inbred introgression lines between $D$. simulans and $D$. sechellia, and to compare our results to QTL studies in D. melanogaster in order to provide some insight on whether the loci accounting for morphological variation within a species are the same as those causing the divergence between species, another important subject in evolutionary biology.

\section{MATERIAL AND METHODS}

\section{Interspecific introgression lines and molecular markers}

Introgression lines were constructed by Dermitzakis et al. (2000) through the introgression of $D$. sechellia into $D$. simulans genome, as follows: $D$. simulans females (wild-caught line "sim2"; Winters, CA) were crossed with D. sechellia males (line "3588"; Drosophila Species Stock Center, 14021-0248.4). F1 females were backcrossed to $D$. simulans males and the backcrossed male progeny was backcrossed to $D$. simulans females. Two hundred and twenty-one lines were then established, each through single-pair mating of siblings from the second backcross. To approach homozygosis, 14 generations of single-pair sib-mating were performed for each line. Several lines were lost, mostly because of reduced viability/fertility. Here, 130 lines were analyzed. The expected proportions of autosomal genome and X-chromosome derived from $D$. sechellia are respectively $1 / 8$ and $1 / 6$, if genes are strictly neutral on viability and fertility. The Y-chromosome is entirely from $D$. simulans.

The molecular map was established by Dermitzakis et al. (2000) and Civetta et al. (2002). These authors estimated the cytological and genetic positions of most markers and also scored the introgression lines according to parental genotypes at each marker. Other markers were latter included by Civetta and Cantor (2003) and Civetta et al. (2005). In total, we used 27 markers (Table 1); for details on primers and polymerase chain reaction (PCR) conditions, see the above-referenced studies. The chromosomes of $D$. simulans, $D$. sechellia and $D$. melanogaster are essentially homosequential (Ashburner et al., 2005), except for an inversion on chromosome 3 of $D$. simulans and D. sechellia $(\operatorname{In}(3 R) 84 F 1$;93F6-7), relative to the standard arrangement of $D$. melanogaster (Ranz et al., 2007). Using FlyBase (http://flybase.bio.indiana. edu/), we reviewed and confirmed the genetic positions of all markers except for marker 24 
(AC001655), which does not fall within the inversion $\operatorname{In}(3 R) 84 F 1$;93F6-7. Its position was corrected following the procedure of Civetta et al. (2002) and is reported in Table 1. Marker 25 (сро) is the only marker located within this inversion.

\begin{tabular}{|c|c|c|c|c|c|c|}
\hline $\mathrm{Chr}$ & Marker & Gene symbol & Cytological $\mathrm{mel}$ & Genetic $m e l(\mathrm{cM})$ & Genetic IG (cM) & Marker diff. \\
\hline \multirow[t]{7}{*}{$\mathrm{X}$} & 1 & w & $3 \mathrm{C} 2$ & 11.5 & 3.6 & 1 \\
\hline & 2 & $C d k 7$ & $4 \mathrm{~F} 1-2$ & 11.0 & 8.9 & 1 \\
\hline & 3 & rux & $5 \mathrm{D} 5$ & 15.0 & 11.2 & 2 \\
\hline & 4 & $\mathrm{sev}$ & $10 \mathrm{~A} 2-4$ & 33.4 & 23.7 & 1 \\
\hline & 5 & $\operatorname{sog}$ & $13 \mathrm{E} 3-8$ & 53.0 & 42.7 & 1 \\
\hline & 6 & Sh & 16E4-F1 & 57.6 & 49.0 & 1 \\
\hline & 7 & $\operatorname{shakB}$ & $19 \mathrm{E} 3$ & 64.0 & 57.9 & 1 \\
\hline \multirow[t]{9}{*}{2} & 8 & aop & 22C3-D1 & 12.0 & 13.0 & 1 \\
\hline & 9 & $A c p 26 A b$ & $26 \mathrm{~A} 5$ & 18.0 & 22.0 & 3 \\
\hline & 10 & ninaC & $27 F 5-6$ & 22.0 & 28.5 & 1 \\
\hline & 11 & $d a$ & 31D11-E1 & 41.3 & 47.8 & 1 \\
\hline & 12 & $\mathrm{Su}(H)$ & $35 \mathrm{~B} 10-\mathrm{C} 1$ & 50.5 & 64.0 & 1 \\
\hline & 13 & $\mathrm{cad}$ & $38 \mathrm{E} 5-6$ & 54.0 & 71.9 & 1 \\
\hline & 14 & mam & $50 \mathrm{C} 23-\mathrm{D} 3$ & 70.3 & 100.5 & 1 \\
\hline & 15 & $A m y-d$ & $53 \mathrm{~F} 13-54 \mathrm{~A} 2$ & 77.9 & 115.3 & 2 \\
\hline & 16 & AC 004365 & 58A4-B1 & 107.6 & 146.5 & 1 \\
\hline \multirow[t]{11}{*}{3} & 17 & rhob & $62 \mathrm{~A} 2$ & 0.2 & 5.0 & 1 \\
\hline & 18 & Cdc37 & $62 \mathrm{~B} 4$ & 5.0 & 8.8 & 1 \\
\hline & 19 & Acp63F & $63 \mathrm{~F} 1$ & 9.0 & 11.9 & 3 \\
\hline & 20 & ple & $65 \mathrm{C} 3$ & 18.0 & 20.3 & 1 \\
\hline & 21 & LanB2 & 67B10 & 28.0 & 30.8 & 1 \\
\hline & 22 & DM22F11T & 73A1-B7 & 44.0 & 56.7 & 1 \\
\hline & 23 & Cat & 75D7-E1 & 47.0 & 71.7 & 2 \\
\hline & 24 & AC001655 & $84 \mathrm{C} 1-4$ & 48.0 & 84.1 & 1 \\
\hline & 25 & сро & 90C10-D1 & 62.0 & 97.0 & 2 \\
\hline & 26 & pnt & 94E11-F1 & 79.0 & 141.5 & 1 \\
\hline & 27 & Anp & 99D5 & 100.0 & 173.6 & 3 \\
\hline
\end{tabular}

Cytological and Genetic refer to the position of each marker; genetic positions are in centiMorgans (cM). mel codes for Drosophila melanogaster and IG codes for D. simulans/D. sechellia introgression lines. Marker diff: parental genotypic differences scored for each marker; where 1 codes for microsatellite repeats, 2 for insertions/deletions and 3 for changes in restriction sites. Marker 25 is located within the inversion $\operatorname{In}(3 R) 84 F 1 ; 93 F 6-7$ of $D$. simulans $/ D$. sechellia hybrids relative to the standard arrangement of chromosome 3 in D. melanogaster.

\section{Phenotypic scoring}

From each introgression line, we randomly selected three inseminated females for the establishment of two oviposition batches in cornmeal sucrose medium at $16.5^{\circ} \mathrm{C}$; each oviposition lasted for two days. From the progeny of each line at each batch, two males and two females had their left wings mounted in distilled water on glass slides. Thus, eight individuals were scored per introgression line, totaling 1040 individuals. For the parental lines, 105 fixed individuals (58 of D. simulans and 47 of $D$. sechellia) were kindly provided by Dr. Andrew Clark, from stocks maintained on cornmeal sucrose medium at $25.0^{\circ} \mathrm{C}$. Their left wings were also mounted on slides for phenotypic scoring. All crosses and measurements were performed in 2001.

Using a least square procedure, the general equation of the ellipse was solved for the Cartesian coordinates of 20 landmarks taken throughout the wing contour (Klaczko and BitnerMathé, 1990; Matta and Bitner-Mathé, 2004), a procedure that allows the fitting of an ellipse 
to the outline of each wing (Figure 1). A wing size estimate (SI) was obtained through the geometric mean between the two ellipse radii $a$ and $b(\mathrm{SI}=\sqrt{\mathrm{ab}})$; SI corresponds to the radius of a circle with the same ellipse area (Klaczko and Bitner-Mathé, 1990). Two aspects of shape were also analyzed: 1$)$ the outline shape of the wing $(\mathrm{SH})$, which was estimated through the ratio $b / a$, and 2) the position of wing veins, each obtained by the angle $(\theta)$ produced between the major ellipse axis and the line that joins the respective landmark to the origin of the ellipse (Figure 1). Although wing size, outline shape and veins position might be biologically correlated, the estimates of SI, SH and $\theta$ angles are algebraically independent of each other. SI and SH express different ellipse properties, as the ellipse size might change without changing its outline shape, and vice-versa. And SH accounts for a possible variation in the wing outline that is not necessarily restricted to variation in veins position.

\section{QTL mapping}

QTLs were mapped through composite interval mapping (CIM) (Zeng, 1993, 1994) using Windows QTL Cartographer 2.5 (Wang et al., 2007). CIM tests the likelihood that a given interval has a QTL affecting the trait compared to the likelihood of the null hypothesis (no QTL), named likelihood ratio (LR). Since some residual variance of non-target QTLs might interfere with the statistical testing at each interval, different numbers of markers can be used as cofactors to control the genetic background (Zeng, 1993). Increasing this number generally results in a higher precision of estimating QTL positions and effects; on the other hand, it reduces the power of finding the QTL (Zeng, 1994).

Here, LR was tested every $2 \mathrm{cM}$ with a flanking window size of $10.0 \mathrm{cM}$. CIM was first performed using all 27 markers (CIM27) as cofactors to control the background variance (model I; Zeng, 1994). To balance between precision and efficiency, a forward regression step was included so that statistically relevant cofactors for each wing trait could be identified within a set of 20 or 15 markers (CIM20 or CIM15) (model IV; Wang et al., 2007). For each wing trait, CIM27, CIM20 and CIM15 were performed by sex (females, males) and for both sexes together (total), in this case using sex as a covariate. LR significance thresholds at $\alpha=0.05$ were set through 1000 permutations of the original phenotypic data. To account for multiple comparisons without being too conservative, results at $\alpha=0.01$ are also presented.

Given that the introgression lines were constructed via a recombinant inbred design derived by sib mating, only additive effects could be estimated. Drosophila simulans was arbitrarily set as the 'high' parental species. Consequently, QTL peaks have positive additive effects when the average phenotypic value of the introgression lines carrying the $D$. simulans allele at the QTL position is greater than the average phenotypic value of lines carrying the respective $D$. sechellia allele; conditioned on background markers and sex covariance. The contrary is true for QTL peaks with negative effects.

\section{Statistical analyses}

For each wing trait, the difference between parental species was estimated as the average phenotypic value of $D$. simulans (the arbitrary 'high' species) minus the average phenotypic value of $D$. sechellia and then converted to standard deviation (SD) units. Sex differ- 
ences were estimated as the average value of females minus the average value of males and also transformed to SD units.

Correlations among wing traits were summarized through principal component analyses (PCA). The coefficients (eigenvectors) of the principal components (PC) estimate the correlation between each original trait and each PC. Eigenvector squares $\left(r^{2}\right)$ represent the fraction of trait variance explained by the PC. For each analysis, 1000 bootstrap resamplings of the original variables were performed. Eigenvectors means and standard errors (SE) were then calculated. The eigenvalues, which represent the fraction of total normalized variance independently explained by each PC, were also estimated.

Regarding the introgression lines, the average between the two individuals of the same sex within each batch was estimated before all statistical analyses; sample size was thus reduced to 520 mean values. For PC analyses and QTL mapping, the average between the batches was also estimated, so that only one mean value per sex per introgression line was used, totaling 260 mean values (130 from each sex).

Basic statistics, ANOVA and PCA were performed with SYSTAT 10.0 (Copyright $^{\circ}$ SPSS Inc., 2000). When multiple comparisons were made, significance levels were adjusted with standard Bonferroni's correction ( $\alpha$ / number of tests). Mantel's test (1967) was carried out with NTSYS-pc 1.70 (Rohlf, 1992).

\section{RESULTS}

\section{Ellipse adjustment and basic statistics}

By solving the ellipse equation for the $x$-observed values, a correlation $(r)$ between the observed and expected $y$-values was calculated for every individual wing. All $r$ values were close to one $(r>0.993$; $\mathrm{SE}<0.0002)$, suggesting that an ellipse was accurately fitted to the outline of each wing (see Figure 1).

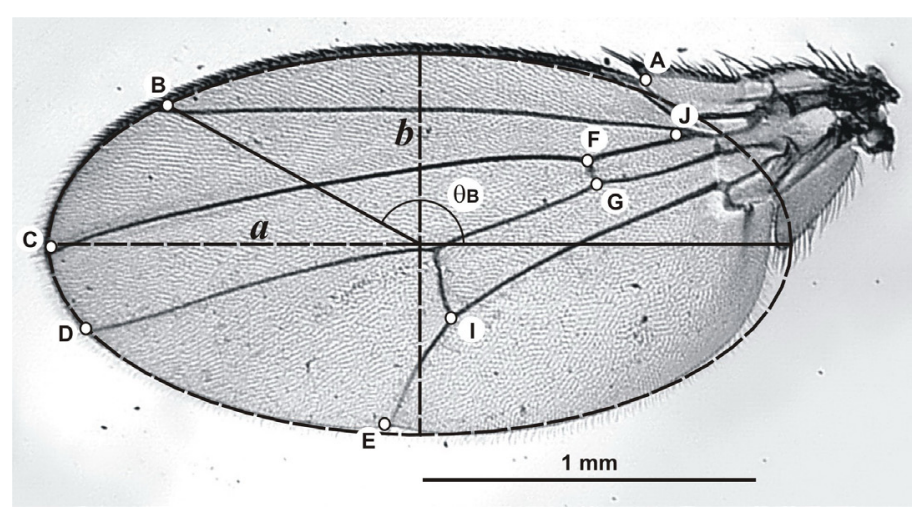

Figure 1. Wing traits and the ellipse adjustment for a Drosophila simulans/D. sechellia introgression female wing. $a$ and $b$ are the two ellipse radii. A, B, C, D, E, F, G, I, and J correspond to the landmarks (open circles) that were marked at intersections between wing veins. B, C, D, and E mark the distal location of longitudinal veins L2, L3, L4, and L5, respectively. The position of each landmark is estimated by the angle between the major ellipse axis and the line that joins the ellipse origin to the given landmark, as shown for the position of B through angle $\theta_{\mathrm{B}}$. 
Wing traits of parental species and introgression lines were normally distributed (Kolmogorov-Smirnov: all $\mathrm{P}>0.05$; except for $\theta_{\mathrm{F}}$ and $\theta_{\mathrm{G}}$ in introgression females). In each case, $\mathrm{SD}$ and coefficient of variation did not differ between the sexes $(F$-test: all $\mathrm{P}>0.05)$. Even when comparing the introgression lines to the parental species, SD and coefficient of variation did not differ ( $F$-test: all $\mathrm{P}>0.05$; except for $\theta_{\mathrm{I}}$ in $D$. simulans $\times$ introgression lines). Statistical analyses and QTL mapping performed for $\mathrm{SH}$ were also performed for $\log (\mathrm{SH})$, a common transformation for ratios between linear measurements. Since the results were nearly identical, only the non-transformed SH analyses are shown.

\section{Wing morphology in the parental species}

The wings of $D$. simulans are larger (bigger SI) than those of $D$. sechellia, but the outline shape of the wing (SH) does not differ significantly (Figure 2 and ANOVA in Table 2). Apart from ANOVA, Table 2 shows the average difference between species for each wing trait, in SD units. Traits with positive values are on average bigger in D. simulans, such as SI, $\theta_{\mathrm{B}}, \theta_{\mathrm{D}}, \theta_{\mathrm{E}}, \theta_{\mathrm{I}}, \theta_{\mathrm{J}}$ and the two ellipse radii $a$ and $b$; the contrary holds for traits with negative values $\left(\theta_{\mathrm{A}}\right.$ and $\left.\theta_{\mathrm{F}}\right)$.

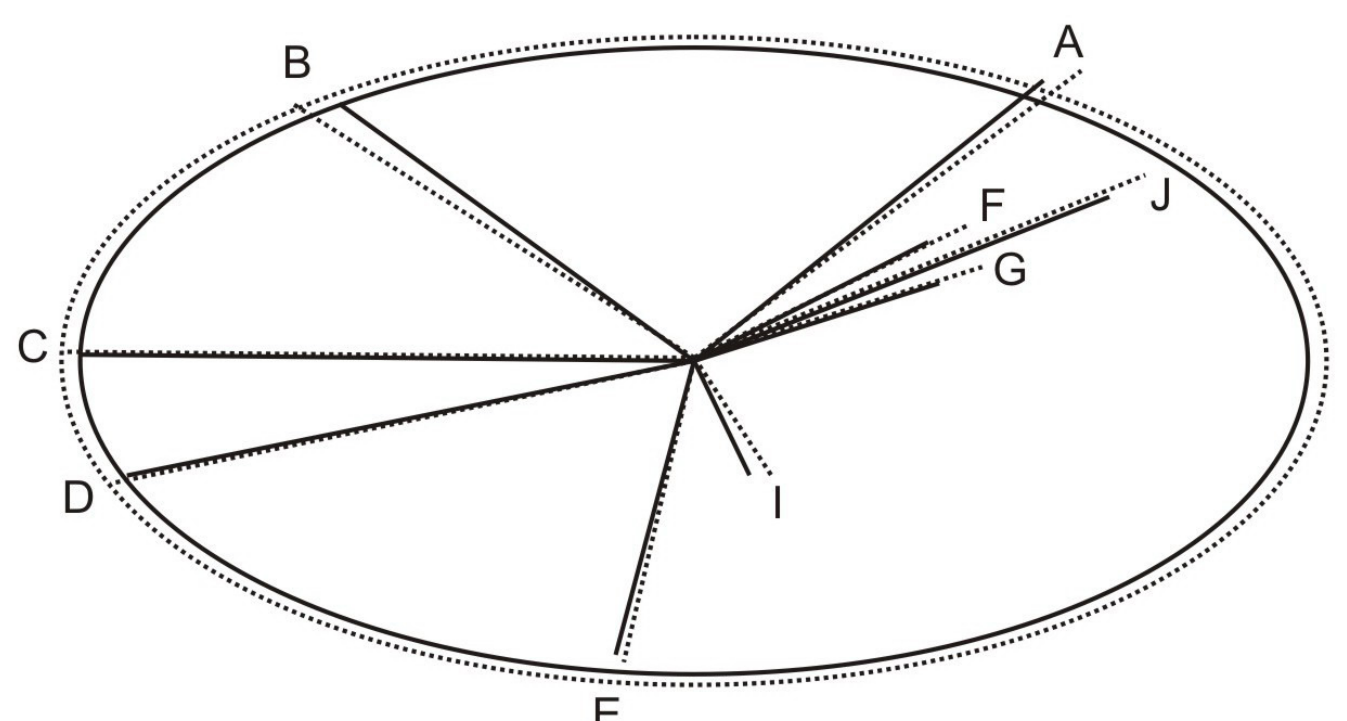

Figure 2. Mean phenotypic values of wing traits in each parental species. Drosophila simulans: dotted lines; $D$. sechellia: solid lines. Ellipses were drawn using the average values of the two ellipse radii $(a$ and $b)$.

The effects of sex and species-sex interaction were also tested through ANOVA (Table 2 ) and are shown in Figure 3. The average differences between the sexes, in SD units, are also presented in Table 2. It follows that female wings are significantly larger and longer than male wings (positive sex diff. for SI and negative for SH), regardless of the species (see Figure 3). Also, the angles $\theta_{\mathrm{B}}, \theta_{\mathrm{C}}$ and $\theta_{\mathrm{F}}$ are on average bigger in females (positive sex diff.), while $\theta_{\mathrm{D}}$, $\theta_{\mathrm{E}}$ and $\theta_{\mathrm{I}}$ are on average smaller (negative sex diff.). Species-sex interaction was found for $\theta_{\mathrm{A}}$, $\theta_{\mathrm{B}}, \theta_{\mathrm{C}}$, and $\theta_{\mathrm{F}}$. 


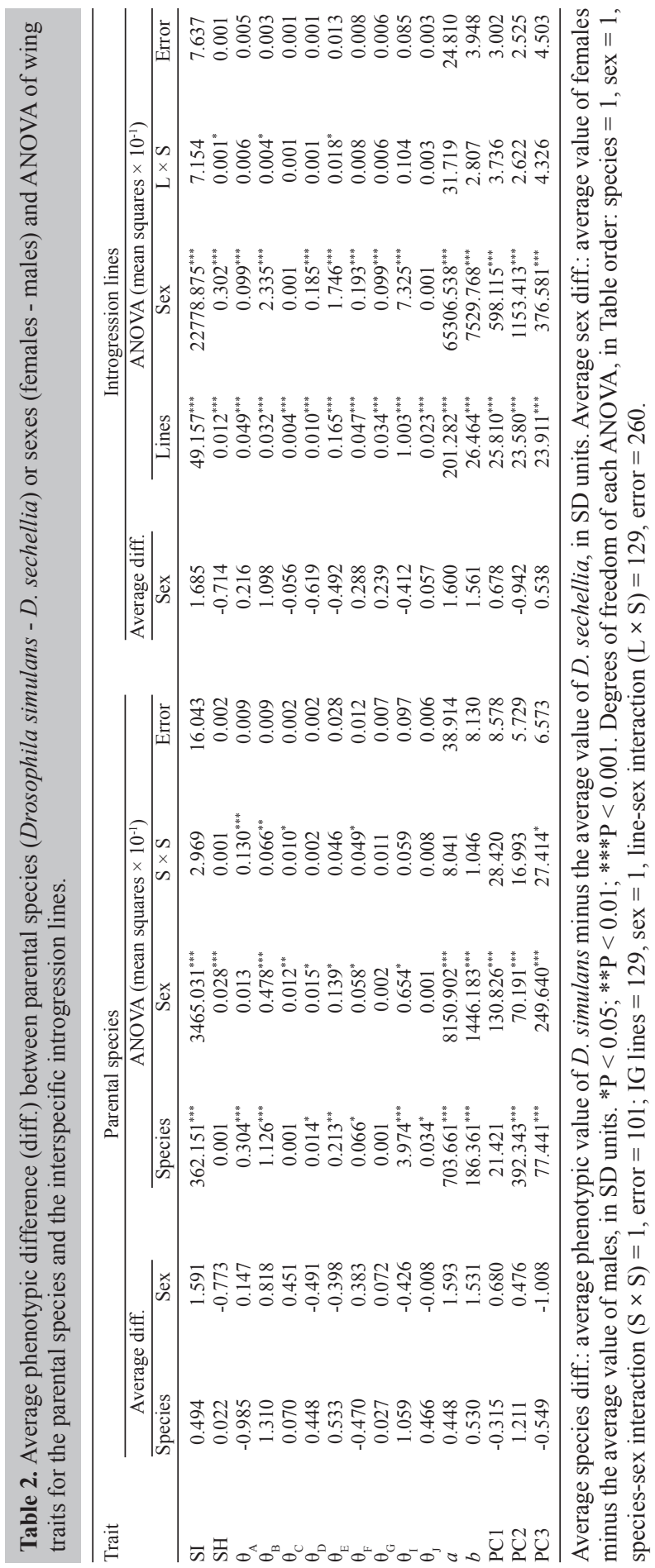



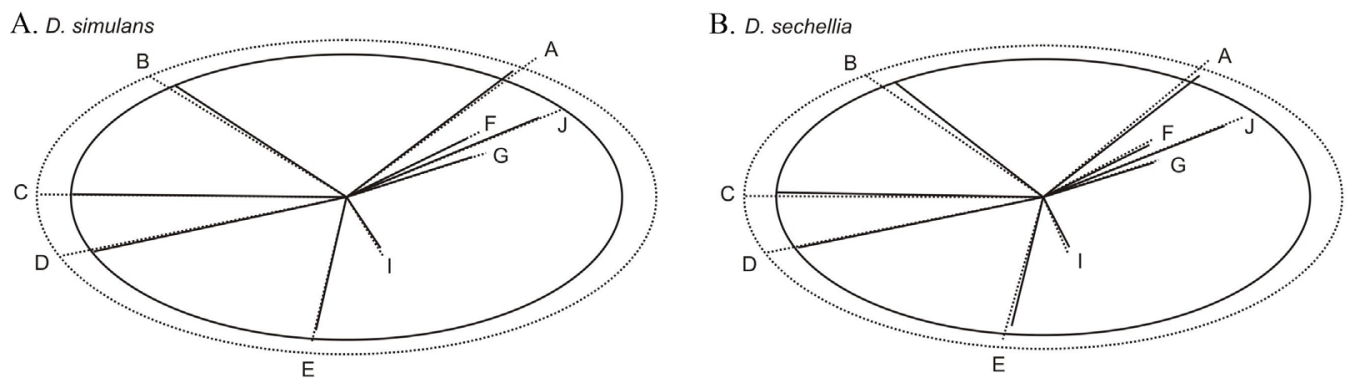

Figure 3. Mean phenotypic values of wing traits by sex. A. Drosophila simulans. B. D. sechellia. Females: dotted lines; males: solid lines.

A PCA was performed to summarize the phenotypic correlations among wing traits (Table 3). PC1 was positively correlated with SI $\theta_{\mathrm{B}}$ but negatively correlated with $\mathrm{SH}$ and the angles $\theta_{\mathrm{D}}, \theta_{\mathrm{E}}$ and $\theta_{\mathrm{I}}$. A considerable fraction of the normalized variance of these traits was explained by PC1 $\left(0.159<r^{2}<0.724\right)$. Hence, PC1 accounts for a pattern of phenotypic correlation in which larger wings (bigger SI) are also longer (lower SH), and the longitudinal veins L2, L4 and L5 are closer together at the distal portion of the wing (bigger $\theta_{\mathrm{B}}$, smaller $\theta_{\mathrm{D}}, \theta_{\mathrm{E}}$ and $\theta_{\mathrm{I}}$ ). Both PC1 and PC3 are primarily associated with sex differences (ANOVA in Table 2) and together they explain $53.3 \%$ of the normalized variance of all wing traits (Table 3). PC3 accounts for an independent but similar pattern: though the eigenvectors of SI, SH and the angles $\theta_{\mathrm{B}}, \theta_{\mathrm{D}}, \theta_{\mathrm{E}}$, and $\theta_{\mathrm{I}}$ have the opposite direction in relation to $\mathrm{PC} 1$, the correlation among these traits was maintained - SI and $\theta_{\mathrm{B}}$ are positively correlated to each other and negatively correlated to $\mathrm{SH}$ and the angles $\theta_{\mathrm{D}}, \theta_{\mathrm{E}}$ and $\theta_{\mathrm{I}}$. In turn, $\mathrm{PC} 2$ is mostly associated with differences between the parental species (ANOVA in Table 2). This PC accounts for a significant proportion of the normalized variance of SI, $\theta_{\mathrm{B}}, \theta_{\mathrm{C}}$, and $\theta_{\mathrm{D}}\left(0.188<r^{2}<0.356\right)$ and these traits are all positively correlated with each other (Table 3 ). In the correlation pattern summarized by PC2, larger wings have the distal portions of veins L2, L3 and L4 located towards a counterclockwise position (bigger $\theta_{\mathrm{B}}, \theta_{\mathrm{C}}$ and $\theta_{\mathrm{D}}$ ).

\section{Wing morphology in the interspecific introgression lines}

The effects of line, sex and line-sex interactions were tested through ANOVA (Table 2). Differences among the introgression lines significantly affected all wing traits, including those that did not differ between the parental species $\left(\mathrm{SH}, \theta_{\mathrm{C}}\right.$ and $\left.\theta_{\mathrm{G}}\right)$.

Sex differences in the introgression lines were similar to those observed in the parental species (see Figure 3) and are presented in Table 2. Indeed, for most wing traits the average sex differences of introgression lines did not differ significantly from the average sex differences of parental species ( $F$-test: all $\mathrm{P}>0.05$; except for $\theta_{\mathrm{C}}, \theta_{\mathrm{G}}$ and $\left.\theta_{\mathrm{J}}\right)$. In the introgression lines, female wings are also bigger and longer than male wings, and the angles $\theta_{\mathrm{B}}, \theta_{\mathrm{F}}$ and $\theta_{\mathrm{G}}$ are significantly bigger in females, while $\theta_{\mathrm{D}}, \theta_{\mathrm{E}}$ and $\theta_{\mathrm{I}}$ are significantly smaller. $\theta_{\mathrm{C}}$ and $\theta_{\mathrm{J}}$ did not differ between the sexes and line-sex interaction was only found for $\mathrm{SH}, \theta_{\mathrm{B}}$ and $\theta_{\mathrm{E}}$ (ANOVA in Table 2).

A PCA was also performed for the introgression lines (Table 3). The first three PC are primarily associated with sex differences, but they are also related to differences among the 
introgression lines (ANOVA in Table 2). While PC1 presents the same correlation pattern as the first PC of parental species, PC2 and PC3 are inversely related when comparing the parental species to the introgression lines (Table 3), as follows. PC2 in the introgression lines shows the same pattern of trait correlations as PC 3 in the parental species. In turn, PC3 in the introgression lines presents a positive correlation to SI, $\theta_{\mathrm{B}}, \theta_{\mathrm{C}}$, and $\theta_{\mathrm{D}}$ as does PC2 in the parental species; regarding the remaining wing traits, less than $13 \%$ of their normalized variance was explained by each of these PC $\left(0.001<r^{2}<0.127\right)$. When a swap-like relationship between PC occurs, it can be caused essentially through a change in the amount of normalized variance explained by each PC (eigenvalues). Here, a possible swap between PC2 and PC 3 might be corroborated by their average sex differences, which are also inversely related between introgression lines and parental species (Table 2). In addition, the total matrix of phenotypic correlation $\left(r_{\mathrm{p}}\right)$ among wing traits in the introgression lines did not differ from the total matrix for the parental species (Mantel's test: $R=0.927$; $>0.05$; matrices are not shown).

\begin{tabular}{|c|c|c|c|c|c|c|}
\hline \multirow[t]{2}{*}{ Trait } & \multicolumn{3}{|c|}{ Parental species } & \multicolumn{3}{|c|}{ Introgression lines } \\
\hline & PC1 & PC2 & PC3 & PC1 & $\mathrm{PC} 2$ & $\mathrm{PC} 3$ \\
\hline SI & 0.519 & 0.433 & -0.501 & 0.517 & -0.411 & 0.276 \\
\hline SH & -0.619 & 0.232 & 0.322 & -0.654 & 0.512 & -0.206 \\
\hline$\theta_{\mathrm{A}}$ & 0.693 & -0.278 & 0.385 & 0.833 & 0.384 & -0.055 \\
\hline$\theta_{B}^{A}$ & 0.399 & 0.574 & -0.480 & 0.755 & -0.289 & 0.429 \\
\hline$\theta_{\mathrm{C}}^{\mathrm{B}}$ & 0.200 & 0.596 & 0.285 & 0.043 & 0.329 & 0.868 \\
\hline$\theta_{\mathrm{D}}^{c}$ & -0.443 & 0.567 & 0.398 & -0.625 & 0.498 & 0.426 \\
\hline$\theta_{\mathrm{F}}^{\mathrm{D}}$ & -0.851 & 0.271 & 0.180 & -0.869 & 0.220 & 0.037 \\
\hline$\theta_{\mathrm{F}}^{\mathrm{E}}$ & 0.875 & 0.162 & 0.247 & 0.861 & 0.380 & -0.095 \\
\hline$\theta_{\mathrm{G}}^{\mathrm{F}}$ & 0.772 & 0.205 & 0.279 & 0.854 & 0.372 & -0.110 \\
\hline$\theta^{\mathrm{G}}$ & -0.732 & 0.356 & 0.217 & -0.796 & 0.151 & 0.044 \\
\hline$\theta_{1}$ & 0.615 & 0.352 & 0.382 & 0.800 & 0.522 & -0.141 \\
\hline Eigenvalues & 4.528 & 1.719 & 1.338 & 5.851 & 1.646 & 1.286 \\
\hline Variance $(\%)$ & 41.167 & 15.626 & 12.160 & 53.195 & 14.960 & 11.689 \\
\hline
\end{tabular}

All eigenvectors were significant (all $\mathrm{P}<0.001$ and $\mathrm{SE} \leq 0.008$; see Methods). Eigenvectors squares $\left(r^{2}\right)$ represent the fraction of the trait variance that is explained by the PC; when $r^{2} \geq 15 \%$, eigenvectors are shown in bold. Variance (\%): percentage of total variance explained by the PC; each of the remaining PC (not shown) explained less than $10 \%$ of total variance.

\section{QTL mapping}

QTL peaks mapped in at least two of the three background analyses (CIM27, CIM20 or CIM15) were considered as significant and are reported in Table 4. Alternatively, peaks found in only one background analysis were considered as marginally significant and are not presented (this additional information is available upon request).

Eighteen significant QTL peaks were identified and are related to 10 original wing traits and two PC (Table 4). The percentage trait variance explained by these peaks $\left(R^{2}\right)$ varied between 5.1 and $74.2 \%$. Significant peaks found for different traits do not necessarily correspond to different QTLs; instead they might represent a single QTL with a pleiotropic effect. Regardless of the sex or trait, peaks associated with the same flanking marker (Mk in Table 4) were initially considered as a single putative QTL (Q1 to Q9 in Table 4). The greatest distance among peaks at a putative QTL was $6.8 \mathrm{cM}$. To test if this distance represents an error associated with the estimation of QTL positions or if it reflects the existence of different but linked 


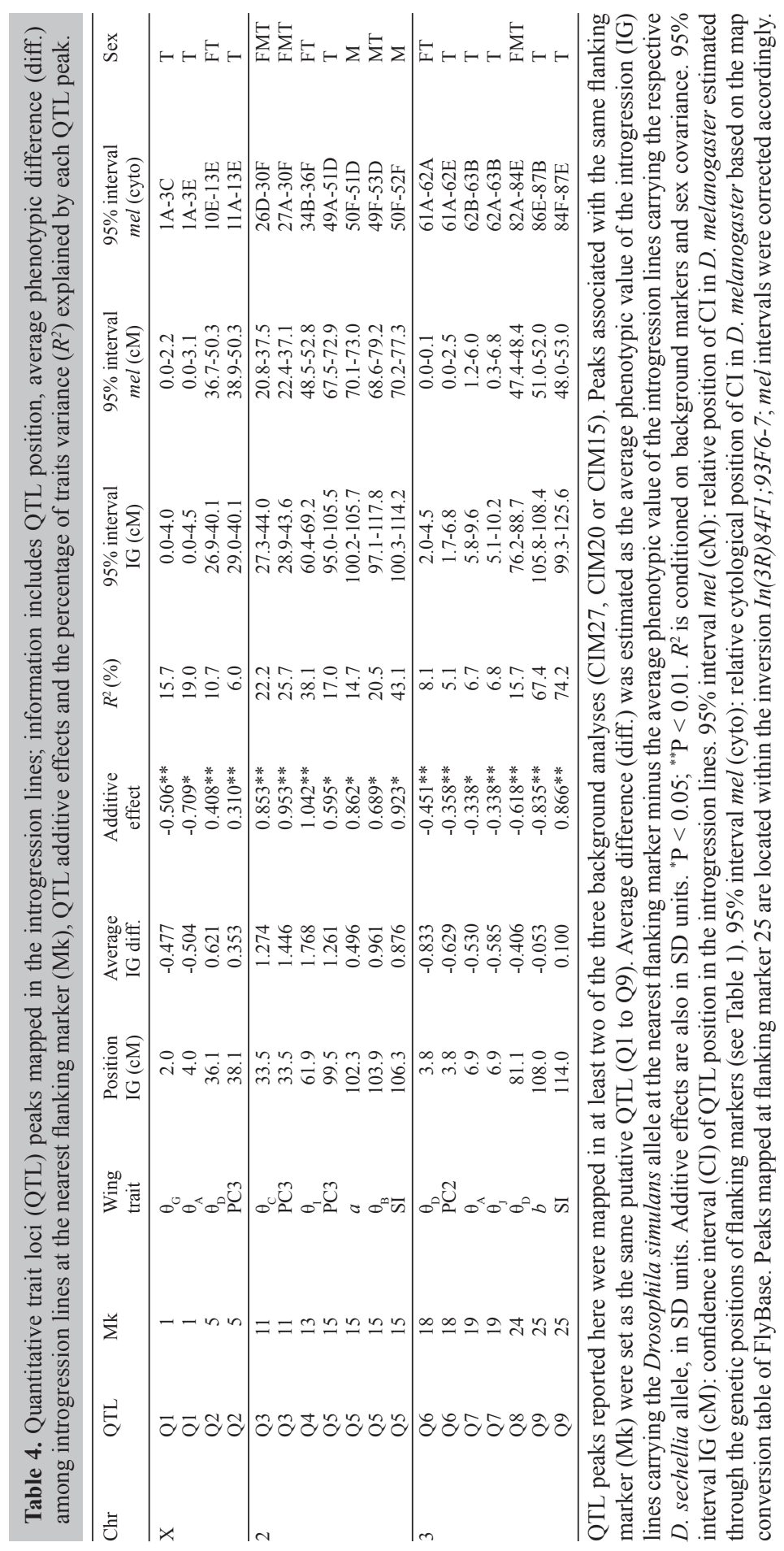


QTLs, multiple-trait mapping (Jiang and Zeng, 1995) was performed. No joint QTL was found for any group of traits assigned as a putative QTL, suggesting that the different peaks at each putative QTL might in fact reflect the existence of different QTLs.

A parallel relationship between the average phenotypic difference of parental species and the average phenotypic difference of introgression lines at QTL peaks was found for SI, $\theta_{\mathrm{A}}, \theta_{\mathrm{B}}, \theta_{\mathrm{I}}, a, \mathrm{PC} 2$, and PC3 (average introgression differences in Table 4 x species differences in Table 2). Taking wing size as an example, SI is bigger in D. simulans relative to $D$. sechellia (positive diff.) and also bigger in the introgression lines carrying $D$. simulans alleles relative to the lines carrying the respective $D$. sechellia allele at the nearest flanking marker of SI QTL peaks (Q5 and Q9; positive diff.). The same might be true for $\theta_{\mathrm{A}}, \theta_{\mathrm{B}}, \theta_{\mathrm{I}}, a, \mathrm{PC} 2$, and $\mathrm{PC} 3$, but the effects are negative in the case of $\theta_{\mathrm{A}}$ and PC2; note that we are comparing the phenotypic differences of PC2 and PC3 with the respective inverted PC in the parental species.

Regarding the remaining QTL peaks, a parallel relationship was not always found. Except for $\theta_{\mathrm{D}}$ in Q2, the average phenotypic differences among introgression lines at QTL peaks for $\theta_{\mathrm{D}}(\mathrm{Q} 6$ and $\mathrm{Q} 8), \theta_{\mathrm{J}}(\mathrm{Q} 7)$ and $b(\mathrm{Q} 9)$ were in the opposite direction from the phenotypic differences between parental species. For instance, the minor ellipse radius (b) is bigger in $D$. simulans relative to $D$. sechellia (positive diff.) but smaller in the introgression lines carrying $D$. simulans alleles relative to the lines carrying the respective D. sechellia allele at the flanking marker of its QTL peak (Q9; negative diff.). Also, QTL peaks were mapped for $\theta_{\mathrm{C}}\left(\mathrm{Q} 3\right.$; positive diff.) and $\theta_{\mathrm{G}}(\mathrm{Q} 1$; negative diff.), even though the phenotypic differences between parental species were not significant for these traits (ANOVA in Table 2).

Additive effects of QTL peaks were estimated through CIM and also transformed to SD units (Table 4). In all cases, additive effects presented the same direction as the respective phenotypic differences at each QTL peak (average diff.). Regarding the magnitude, additive effects were generally smaller but sometimes exceeded the magnitude of phenotypic introgression differences, as in the case of SI (Q5 and Q9), $\theta_{\mathrm{A}}(\mathrm{Q} 1), \theta_{\mathrm{G}}(\mathrm{Q} 1), \theta_{\mathrm{D}}$ (Q8), $a(\mathrm{Q} 5)$, and $b(\mathrm{Q} 9)$.

\section{DISCUSSION}

\section{Genetic basis of interspecific divergence in wing size}

Regarding the quantitative variation in wing size, which might have an adaptive nature, we observed that the wings of $D$. simulans are significantly larger than those of $D$. sechellia. This wing size divergence was already found between these sibling species by Civetta and Singh (1998) and MacDonald (2002). Two of the QTLs mapped in the interspecific introgression lines (Q5 and Q9) were associated to wing size variation in the same direction as the phenotypic difference observed between the parental species.

For this reason, these peaks might reflect the existence of loci that account for at least part of the wing size variation between $D$. simulans and $D$. sechellia. Each peak explains a large fraction of the total wing size variation in the introgression lines $\left(R^{2}\right.$ values in Table 4). But the magnitudes of the additive effects might be overestimated, which is a common problem in QTL mapping experiments. This might be the true for both 
QTL peaks, but especially for Q9 on chromosome 3, since the additive effect of this peak (0.866) greatly exceeded the magnitude of average phenotypic differences among introgression lines with different genotypes at the closest marker locus (0.100). As reviewed by Mackay et al. (2009), overestimation of QTL effects generally occurs when different QTLs with small effects in the same direction happen to be closely linked. Hence, it is possible that each QTL peak mapped for SI accounts for the effects of different loci. It is also likely that other non-mapped QTLs with smaller additive effects in the opposite direction of Q5 and Q9 contribute to generate the amplitude of wing size divergence found in the introgression lines, given that several loci might be involved in the determination of wing size (Carreira et al., 2009). A further investigation of the genetic basis of wing size variation between these sibling species would greatly benefit from increasing the number of markers and sample sizes, in high-resolution QTL mapping.

In addition, we noted that the putative Q5 QTL, at which an SI peak was mapped, was also associated with the shape trait that gives the positioning of L2 vein $\left(\theta_{\mathrm{B}}\right)$. This is not an unexpected result, since these traits already presented a significant genetic correlation of $36.7 \%$ in D. simulans (Matta and Bitner-Mathé, 2004). Moreover, the QTL peaks for SI and $\theta_{\mathrm{B}}$ found at Q5 were both associated with phenotypic differences in the introgression lines that are oriented in the same direction as the differences observed between $D$. simulans and $D$. sechellia. Therefore, we expect that one or more loci within this QTL region should be related to the genetic control of the correlated variation between wing size and the second longitudinal vein, both within and between species.

After the initial exploratory mapping performed here, a more direct approach would be to search for candidate genes already identified in D. melanogaster intraspecific studies. At least in theory, the loci that account for variation within a species might also be involved in the genetic control of phenotypic divergence among closely related species. In a QTL study in $D$. melanogaster, Zimmerman et al. (2000) identified eight QTLs for wing size variation. Two of them were mapped to cytological intervals $(52 \mathrm{~A}-53 \mathrm{~F}$ and $87 \mathrm{~A}-88 \mathrm{~F})$ that overlap the relative position in D. melanogaster estimated for the 95\% confidence intervals of Q5 and Q9 in this study (50F-52F and 84F-87E, respectively). Similarly, Calboli et al. (2003) found eight QTLs associated with wing area; one of them also spanned the cytological region estimated for Q5, while another mapped very closely to the interval of Q9 (52D-53E and 87F-89B, respectively). Given this clear overlap between intra- and interspecific QTLs, even when comparing different studies, it is possible that genes associated with wing size variation in D. melanogaster may also be related to the genetic control of wing size divergence between $D$. simulans and D. sechellia. A search for such genes should begin within the cytological intervals 50F-53F and $84 \mathrm{~F}-89 \mathrm{~B}$, considering the total overlap among our study and two other mentioned above.

By using this approach, some good candidate genes could be inferred from the results of a recent study. Carreira et al. (2009) identified 92 genes associated with different body size traits in $D$. melagonaster, many of which (68 genes) significantly affected wing size in males or females of $P$-element insertion lines. A $P$-element insertion in the coding region of the gene mastermind (mam; cytological position: 50C23-D3) significantly affected the size of male wings (Supplementary material of Carreira et al., 2009), and this gene is closely located to the cytological interval of Q5. Coincidently, this wing size QTL peak was found only in introgression males (see Sex in Table 4). mam is related to the processes of asymmetric cell division and morphogenesis of wing imaginal disc, including the formation of wing margin 
and the adhesion of dorsal and ventral wing surfaces (Yedvobnick et al., 2004). Similarly, two genes identified in the study of Carreira et al. (2009) are located near the region of Q9, which was mapped for both males and females in the introgression lines. The non-coding region of the gene forkhead box, sub-group $O$ (foxo; cytological position: 88A5-A7) was affected by a $P$-element insertion, which caused a significant difference in the size of female wings relative to a control line. In turn, an insertion in the coding region of gene $p x b$ (cytological position: 89A1-A2) significantly affected wing size in males. foxo is directly related to the regulation of the insulin-like growth factor signaling pathway and the cellular processes of growth, proliferation and size, being important for the control of organ size in Drosophila (Kramer et al., 2003). pxb seems to be a putative attenuator of Hedgehog signaling in imaginal discs of Drosophila legs (Inaki et al., 2002); however, the exact function in the wing is unknown. The future goal is to test if these genes are associated with the wing size variation between $D$. simulans and D. sechellia.

Apart from the two additive QTLs found here, other loci should be involved in the control of intra- and interspecific wing size variation in species of the melanogaster subgroup. Eight QTLs related to wing size were found in Zimmerman et al. (2000) and in Calboli et al. (2003), but these QTLs are not necessarily the same in both studies. The genetic effects observed for wing size variation in D. melanogaster are generally dominant (Zimmerman et al., 2000; Gilchrist and Partridge, 2001). The fact that we have identified only two putative QTLs for size traits might be explained by the experimental design of the introgression lines, through which only additive effects could be mapped.

\section{Wing shape and sexual dimorphism}

A recurrent pattern of intraspecific variation among wing traits has been observed in Drosophila species, including D. simulans, and is mostly related to sex, although it might also be affected by other genetic and environmental variation (Bitner-Mathé and Klaczko, 1999b; Matta and Bitner-Mathé, 2004). It follows that longer wings are generally larger and have the second, fourth and fifth longitudinal veins on average closer to each other at the distal region of the wing.

However, this pattern of phenotypic correlation was not observed between the species that we analyzed. We found that $D$. simulans have larger wings and the distal portions of the second, third and fourth longitudinal veins are located towards a counter-clockwise positioning relative to the wings of $D$. sechellia. This result shows that the interspecific wing divergence, as a whole, cannot be explained by a mere quantitative increase in intraspecific differences. This pattern of interspecific variation was summarized by the second principal component (PC2) and is similar to that reported by MacDonald (2002). Genes that lie within the chromosome regions of QTL peaks mapped for PC3 (Q2, Q3 and Q5) in the introgression lines, which corresponds to $\mathrm{PC} 2$ in parental species, are potential candidates for controlling the overall pattern of size and shape divergence between the wings of $D$. simulans and $D$.

sechellia. Nevertheless, a direct identification of candidate genes by searching the literature becomes rather speculative for wing shape traits, since wing shape variation is described in different studies through methods that are difficult to compare.

Another interesting finding was that the wings of $D$. simulans and $D$. sechellia diverged in size without changing the outline shape. Intraspecific studies in different species, 
even from different subgenera such as D. melanogaster and D. mediopunctata, have already suggested that wing size and some aspects of wing shape have different genetic basis (Bitner-Mathé and Klaczko, 1999c; Zimmerman et al., 2000; Gilchrist and Partridge, 2001; Matta and Bitner-Mathé, 2004). Our results support this suggestion with regards to the interspecific variation between two close species, as no QTL was mapped for SH, despite the two found for SI.

Regarding sexual dimorphism, we observed that male wings are on average rounder and smaller than female wings, while the longitudinal veins are more spread apart at the distal tip of the wing; this pattern of phenotypic correlations was found in each parental species (Figure 3), as well as in the introgression lines. This result shows that neither the natural processes of adaptation and drift during the speciation of $D$. simulans and $D$. sechellia, nor the artificial events of hybridization and inbreeding performed during the construction of the introgression lines, were sufficient to break apart the correlations among wing traits. In fact, this sexual dimorphism has already been found in D. melanogaster and D. simulans (Bitner-Mathé and Klaczko, 1999b; Matta and Bitner-Mathé, 2004). Gilchrist et al. (2000) also observed that 'male wings are shorter and broader than female wings across all populations', even when the $D$. melanogaster populations were derived from the extreme ends of latitudinal clines or from different continents. Given this remarkable similarity across populations, the authors suggested the existence of a developmental constraint attributable to the sexual size dimorphism; a constraint that could limit the range of wing shape variation between genders in $D$. melanogaster. Our results corroborate and extend this hypothesis to another species of the melanogaster subgroup, D. sechellia. One QTL peak (Q6) was associated with the principal component that summarizes this pattern (PC2 in the introgression lines). So it is possible that this peak indicates the location of one or more loci associated with this sexual dimorphism. But, because of the different methods for describing the variation in wing shape traits, a direct identification of candidate genes within the cytological region of this QTL peak (61A-62E in D. melanogaster) might not be trivial. In this case, high-resolution mapping or complementation tests should be more promising in the search for candidate genes that control the sexual dimorphism of wing size and shape traits in different species.

It should be noted that, for each QTL mapped in this study, we only infer a possible genetic control of interspecific differences when other studies corroborate the species differences observed here, as in the case of wing size (Civetta and Singh, 1998; MacDonald, 2002) or the above-mentioned patterns of interspecific variation (MacDonald, 2002) and sexual dimorphism (Bitner-Mathé and Klaczko, 1999b; Matta and Bitner-Mathé, 2004). This is because we were not able to raise the parental species under the same conditions as the mapping introgression lines, especially temperature $\left(16.5^{\circ} \mathrm{C}\right.$ for the introgression lines; $25.0^{\circ} \mathrm{C}$ for the parental species). But the results of QTL mapping in the introgression lines do not change; what could change is the interpretation of the QTL effects, which are based on the comparison with the phenotypic differences found between the parental species. For this reason, we only assume a putative role of a given QTL in controlling the interspecific divergence of wing traits between $D$. simulans and $D$. sechellia when these interspecific differences were also observed by other investigators. But we bear in mind that the putative interspecific effect of each QTL and candidate gene will need to be validated in future studies.

On the other hand, the QTL found in our study could be related to some degree of transgressive segregation, i.e., when the phenotypic differences among hybrid individuals (or 
hybrid lines) are greater or novel relative to the parental lines. Transgressive segregation is caused by interaction and complementation among different loci for the same quantitative trait and is also an interesting phenomenon from the standpoint of evolutionary biology, since it might be associated with adaptive radiation in different organisms (reviewed in Rieseberg et al., 2003). In QTL mapping experiments, the result of transgressive segregation is that the phenotypic differences of hybrid lines (introgression lines in our case) will show greater magnitude or opposite direction in relation to the differences between parental species. This seems to be the case for the QTL peaks associated with the wing shape traits $\theta_{\mathrm{D}}\left(\mathrm{Q} 6\right.$ and Q8), $\theta_{\mathrm{J}}(\mathrm{Q} 7)$ and $b$ (Q9), for which a parallel relationship between the phenotypic differences of introgression lines and parental species was not observed. This might also be the case for $\theta_{C}(Q 3)$ and $\theta_{G}$ (Q1), for which QTL peaks were mapped in the introgression lines but no significant variation was observed between the parental species. Within the cytological regions of these QTLs, one or more loci might account for at least part of the putative transgressive segregation of these traits; a proposition that is also opened for validation in other studies.

Overall, we have identified QTL regions associated with quantitative variation in wing size and shape traits that might be related to interspecific variation between two sibling species of Drosophila or even to transgressive segregation of their hybrids. In either case, this first attempt to map the location of loci involved in species divergence has provided the identification of some candidate genes for the control of quantitative wing size variation in different species, as well as some cytological regions that should help in the quest for finding and characterizing candidate genes associated with interspecific variation or sexual dimorphism in different wing traits, given that these regions might serve as good starting points for where to look.

\section{ACKNOWLEDGMENTS}

We thank A.G. Clark for the introgression lines and the molecular map, as well as for invaluable contributions during the course of this study. We also thank K.L. Montooth, A. Civetta and R. da Matta for help in initial analyses, D. Rodrigues for medium preparation and dedicated laboratory maintenance, and J.R. David for thoughtful comments on earlier drafts. Research supported by Conselho Nacional de Desenvolvimento Científico e Tecnológico (CNPq; BCB-M: \#485332/2007-8) and Fundação de Amparo à Pesquisa do Rio de Janeiro (FAPERJ; BCB-M: \#E-26/171.314/2008) and was part of B.P. Matta's PhD research in Genetics, at the Department of Genetics of the Universidade Federal do Rio de Janeiro. B.P. Matta received a graduate scholarship from CNPq.

\section{REFERENCES}

Ashburner M, Golic KG and Hawley RS (2005). Drosophila: a Laboratory Handbook. 2nd edn. Cold Spring Harbor Laboratory Press, New York.

Atkinson D and Sibly RM (1997). Why are organisms usually bigger in colder environments? Making sense of a life history puzzle. Trends Ecol. Evol. 12: 235-239.

Bitner-Mathé BC and Klaczko LB (1999a). Size and shape heritability in natural populations of Drosophila mediopunctata: temporal and microgeographical variation. Genetica 105: 35-42.

Bitner-Mathé BC and Klaczko LB (1999b). Plasticity of Drosophila melanogaster wing morphology: effects of sex, temperature and density. Genetica 105: 203-210.

Bitner-Mathé BC and Klaczko LB (1999c). Heritability, phenotypic and genetic correlations of size and shape of 
Drosophila mediopunctata wings. Heredity 83: 688-696.

Bochdanovits Z and De Jong G (2003). Experimental evolution in Drosophila melanogaster: interaction of temperature and food quality selection regimes. Evolution 57: 1829-1836.

Calboli FC, Kennington WJ and Partridge L (2003). QTL mapping reveals a striking coincidence in the positions of genomic regions associated with adaptive variation in body size in parallel clines of Drosophila melanogaster on different continents. Evolution 57: 2653-2658.

Carreira VP, Mensch J and Fanara JJ (2009). Body size in Drosophila: genetic architecture, allometries and sexual dimorphism. Heredity 102: 246-256.

Civetta A and Singh RS (1998). Sex and speciation: genetic architecture and evolutionary potential of sexual versus nonsexual traits in the sibling species of the Drosophila melanogaster complex. Evolution 52: 1080-1092.

Civetta A and Cantor EJ (2003). The genetics of mating recognition between Drosophila simulans and D. sechellia. Genet. Res. 82: 117-126.

Civetta A, Waldrip-Dail HM and Clark AG (2002). An introgression approach to mapping differences in mating success and sperm competitive ability in Drosophila simulans and D. sechellia. Genet. Res. 79: 65-74.

Civetta A, Montooth KL and Mendelson M (2005). Quantitative trait loci and interaction effects responsible for variation in female postmating mortality in Drosophila simulans and D. sechellia introgression lines. Heredity 94: 94-100.

Dermitzakis ET, Masly JP, Waldrip HM and Clark AG (2000). Non-Mendelian segregation of sex chromosomes in heterospecific Drosophila males. Genetics 154: 687-694.

Dworkin I and Gibson G (2006). Epidermal growth factor receptor and transforming growth factor-beta signaling contributes to variation for wing shape in Drosophila melanogaster. Genetics 173: 1417-1431.

Falconer DS and Mackay TFC (1996). Introduction to Quantitative Genetics. 4th edn. Addison Wesley Longman (Pearson Education), Essex.

Gilchrist AS and Partridge L (2001). The contrasting genetic architecture of wing size and shape in Drosophila melanogaster. Heredity 86: 144-152.

Gilchrist GW and Huey RB (2004). Plastic and genetic variation in wing loading as a function of temperature within and among parallel clines in Drosophila subobscura. Integr. Comp. Biol. 44: 461-470.

Gilchrist AS, Azevedo RB, Partridge L and O'Higgins P (2000). Adaptation and constraint in the evolution of Drosophila melanogaster wing shape. Evol. Dev. 2: 114-124.

Hey J and Kliman RM (1993). Population genetics and phylogenetics of DNA sequence variation at multiple loci within the Drosophila melanogaster species complex. Mol. Biol. Evol. 10: 804-822.

Huey RB, Gilchrist GW, Carlson ML, Berrigan D, et al. (2000). Rapid evolution of a geographic cline in size in an introduced fly. Science 287: 308-309.

Inaki M, Kojima T, Ueda R and Saigo K (2002). Requirements of high levels of Hedgehog signaling activity for medialregion cell fate determination in Drosophila legs: identification of pxb, a putative Hedgehog signaling attenuator gene repressed along the anterior-posterior compartment boundary. Mech. Dev. 116: 3-18.

Jiang C and Zeng ZB (1995). Multiple trait analysis of genetic mapping for quantitative trait loci. Genetics 140: 11111127.

Jones CD (2005). The genetics of adaptation in Drosophila sechellia. Genetica 123: 137-145.

Klaczko LB and Bitner-Mathé BC (1990). On the edge of a wing. Nature 346: 321.

Kramer JM, Davidge JT, Lockyer JM and Staveley BE (2003). Expression of Drosophila FOXO regulates growth and can phenocopy starvation. BMC Dev. Biol. 3: 5 .

Lecuit T and Le Goff L (2007). Orchestrating size and shape during morphogenesis. Nature 450: 189-192.

Louis J and David JR (1986). Ecological specialization in the Drosophila melanogaster species subgroup: a case study of D. sechellia. Acta Oecol. 7: 215-229.

MacDonald SJ (2002). Wing shape in the Drosophila simulans species complex. Drosophila Inf. Serv. 85: 31-34.

Mackay TF, Stone EA and Ayroles JF (2009). The genetics of quantitative traits: challenges and prospects. Nat. Rev. Genet. 10: 565-577.

Mantel N (1967). The detection of disease clustering and a generalized regression approach. Cancer Res. 27: 209-220.

Matta BP and Bitner-Mathé BC (2004). Genetic architecture of wing morphology in Drosophila simulans and an analysis of temperature effects on genetic parameter estimates. Heredity 93: 330-341.

Ranz JM, Maurin D, Chan YS, von Grotthuss M, et al. (2007). Principles of genome evolution in the Drosophila melanogaster species group. PLoS Biol. 5: e152.

Rieseberg LH, Widmer A, Arntz AM and Burke JM (2003). The genetic architecture necessary for transgressive segregation is common in both natural and domesticated populations. Philos. Trans. R. Soc. Lond. B. Biol. Sci. 358: 1141-1147.

Rohlf JF (1992). NTSYS-pc Numerical Taxonomy and Multivariate Analysis System. Version 1.70. Applied Biostatistics 
Inc., New York.

Wang S, Basten CJ and Zeng ZB (2007). Windows QTL Cartographer. Version 2.5. Bioinformatics Research Center, Department of Statistics, North Carolina State University, Raleigh.

Weber KE, Johnson N, Champlin D and Patty A (2005). Many P-element insertions affect wing shape in Drosophila melanogaster. Genetics 169: 1461-1475.

Yedvobnick B, Kumar A, Chaudhury P, Opraseuth J, et al. (2004). Differential effects of Drosophila mastermind on asymmetric cell fate specification and neuroblast formation. Genetics 166: 1281-1289.

Zeng ZB (1993). Theoretical basis for separation of multiple linked gene effects in mapping quantitative trait loci. Proc. Natl. Acad. Sci U. S. A. 90: 10972-10976.

Zeng ZB (1994). Precision mapping of quantitative trait loci. Genetics 136: 1457-1468.

Zimmerman E, Palsson A and Gibson G (2000). Quantitative trait loci affecting components of wing shape in Drosophila melanogaster. Genetics 155: 671-683. 